CAHIERS DE

NARRATOLOGIE

\section{Cahiers de Narratologie}

Analyse et théorie narratives

$14 \mid 2008$

Prose d'idées : formes et savoirs

\title{
Enjeux et ressorts de la prose d'idées dans l'Espagne du XVIIe siècle
}

Le cas d'Antonio López de Vega (1586?-1656?)

Marie-Laure Acquier

\section{OpenEdition}

\section{Journals}

Édition électronique

URL : http://journals.openedition.org/narratologie/613

DOI : $10.4000 /$ narratologie.613

ISSN : 1765-307X

\section{Éditeur}

LIRCES

Référence électronique

Marie-Laure Acquier, "Enjeux et ressorts de la prose d'idées dans l'Espagne du XVIle siècle », Cahiers de Narratologie [En ligne], 14 | 2008, mis en ligne le 15 juillet 2010, consulté le 15 novembre 2019. URL : http://journals.openedition.org/narratologie/613

Ce document a été généré automatiquement le 15 novembre 2019.

\section{cc) (†) $\odot$}

Cahiers de Narratologie - Analyse et théorie narratives est mis à disposition selon les termes de la licence Creative Commons Attribution - Pas d'Utilisation Commerciale - Pas de Modification 4.0 International. 


\title{
Enjeux et ressorts de la prose d'idées dans l'Espagne du XVIIe siècle
}

\author{
Le cas d'Antonio López de Vega (1586?-1656?)
}

Marie-Laure Acquier

1 En Espagne, la période baroque, au moins à ses débuts, est marquée par un profond élan de théorisation autour du statut de la littérature. Âge de l'inconstance et du mouvement, le baroque cherche à trouver ou à retrouver des règles afin de comprendre, canaliser, juger, critiquer, délier ou « déplier » dirait Gilles Deleuze, une production textuelle qui ne cesse de croître. Les nouvelles conditions de production du livre et l'adaptation des auteurs aux attentes d'un public élargi ouvrent un vaste champ à l'activité réflexive sur les grandes formes littéraires. Le panorama littéraire s'en trouve profondément affecté.

2 Le théâtre est en plein essor et en pleine évolution sous les coups de butoir de la nouvelle comédie lopesque qui remodèle les préceptes dramaturgiques d'Aristote. L'engagement militant des tenants rigoristes de la poétique aristotélicienne contre ce qui est devenu la "comedia nueva " donne aux débats sur le théâtre des allures de conflit ouvert. Dans l'Art nouveau de faire des comédies, daté de 1605, Lope de Vega revendique pour la première fois le goût du public comme source d'inspiration.

Dans le même temps, les récits de fiction cherchent leurs repères narratifs dans le roman byzantin ${ }^{1}$ qui remet au goût du jour les récits d'Héliodore, dans le roman pastoral qui héroïse l'univers bucolique ${ }^{2}$, dans le roman mauresque qui fait revivre le temps de la «frontière » musulmane $\mathrm{du} \mathrm{Xv}^{\mathrm{e}}$ - l'Histoire de l'Abencerrage, nouvelle incluse dans la Diana de Montemayor reprend les thèmes des romances de la frontière -, dans le roman picaresque surtout, ces récits d'une vie qui obéissent souvent à une construction dialectique et dont les héros ne sont plus que des gueux.

4 C'est dans ce contexte d'évolution et de constitution des genres, de foisonnement générique aussi, qu'il faut replacer l'opérativité de la catégorie de prose d'idées. 
$5 \quad$ Nous le verrons dans un premier temps de notre réflexion - appelons-le théorique - qui sera suivi par l'analyse de certains aspects formels et thématiques de l'œuvre de López de Vega, philosophe moral, poète et prosateur, dans l'Espagne du XVII e siècle espagnol, théoricien du théâtre et critique des disciplines du savoir à ses heures.

\section{Prose d'idées, littérature d'idées}

Quelle est donc l'opérativité de la notion de prose d'idées à l'époque baroque ? On est tentée de dire qu'elle découle de sa grande indétermination. La catégorie apparaît explicitement dans les ouvrages généraux de littérature espagnole et permet de ranger pêle-mêle dans des chapitres séparés des grands genres (théâtre, roman naissant, poésie) une littérature abondante et éclectique. Sa capacité d'englobement, son référent générique: "prose» associée à son ancrage dans le domaine de la connaissance, des savoirs et des idéologies : «idées " permet d'octroyer un chapeau à une production très disparate, dont la variété thématique et générique est d'ailleurs un ressort publicitaire, et qui effectivement jouit à l'époque d'un très grand succès. Nous pourrions emprunter à Stéphane Pujol son expression « littérature d'idées » qu'il donne pour équivalente de celle de "prose d'idées ». Ni dans l'une ni dans l'autre, la fiction ne fait figure d'élément discriminant. L'inventio, mise en avant par Lopez Pinciano dans le système aristotélicien dès le XVI $\mathrm{e}^{\mathrm{e}}$ siècle en Espagne, côtoie sans gêne l'imitatio. Les textes que l'on range dans la catégorie prose d'idées ou littérature d'idées présentent souvent, non systématiquement, un habillage fictionnel. Le dialogue didactique ou philosophique est très souvent dramatisé, le discours à visée scientifique peut émaner de personnages fictifs. Par ailleurs, la fiction par L'exemplum peut parfaitement servir une démonstration visant à la recherche du vrai. L'expression citée par Pujol est en outre intéressante car elle ôte tout référent formel (prose vs poésie) et ne garde que la mention "littérature » au sens large pour désigner une production textuelle où le genre n'est pas discriminant. La période baroque dans son activité réflexive intense sur les genres passe toujours par le prisme du système rhétorique aristotélicien qui traite avant tout des modes d'énonciation (épique, dramatique, lyrique) et par la théorie cicéronienne des styles (simple, moyen, élevé), sans référence aux genres tels que nous les entendons aujourd'hui.

Que trouve-t-on rangé dans les chapitres sur la «prose d'idées », dans nos littératures espagnoles?

\section{Un répertoire d'œuvres à succès}

8 Généralement, dès le titre des chapitres qui lui sont consacrés, la prose d'idées est associée à Baltasar Gracián. Le génie de l'écrivain, son succès international justifient pleinement que le père jésuite incarne à lui seul la catégorie. De fait, les synthèses critiques contenues dans les chapitres s'intéressent essentiellement aux œuvres politiques et morales des grands auteurs du temps passés à la postérité, Diego Saavedra Fajardo, Francisco de Quevedo, Baltasar Gracián. Plus rarement on trouvera trace d'auteurs précurseurs des lumières dans leurs préoccupations comme Gaspar Guttiérez de los Ríos (auteur des 61 discours de El Hombre practico (Bruxelles, 1680) inspirés du cartésianisme mais aussi des œuvres morales de Plutarque ${ }^{3}$ ), d'auteurs dits économiques ou « $\operatorname{arbitristas}^{4} »$, tel le tacitiste Alamos de Barrientos, ou des tenants du 
genre de l'épître comme Espinosa y Malo, ou encore des adeptes du tableau de mœurs et des miscellanées, tel Juan de Zabaleta ${ }^{5}$.

Les auteurs classés sous la catégorie "prose d'idées " sont généralement les grands polygraphes ayant cultivé plusieurs veines littéraires comme la littérature emblématique, politique, morale à travers différentes formes discursives ou narratives, le traité de morale, le dialogue humaniste, l'épître, le songe didactique ou satirique, le pamphlet, la biographie laïque, la chronique. Quevedo est l'exemple même de ce "polygraphisme généralisé ", pour reprendre une formule due à Maurice Molho ${ }^{6}$. L'éclectisme de cette production très abondante, et très variée dans ses thématiques, justifie sa mise à l'écart hors des grandes catégories, théâtre, poésie lyrique, fiction romanesque. Si les littératures ne font état d'aucune définition de la prose d'idées si générale ou imprécise soit-elle, pour le moins ont-elles le mérite de donner à connaître des prosateurs dont les œuvres jouirent à l'époque d'une grande audience nationale et internationale comme en témoignent les nombreuses éditions espagnoles de leurs ouvrages et leurs traductions à l'étranger. Le cas de Gracián traduit par Amelot de la Houssaye étant le plus connu?

10 Néanmoins dans les chapitres des littératures espagnoles que nous venons de décrire brièvement, sont laissés de côté par un choix implicite ou explicite de grands pans de la littérature politique, de la littérature religieuse, littérature hagiographique ou autobiographique, les biographies édifiantes, l'historiographie ou les « canards » - de ce mot qui traduit mal le terme espagnol « relaciones de sucesos » et qui correspond à ces sortes de reportages écrits et très souvent imprimés sur les mariages, les entrées royales, les visites d'ambassadeur ou la vie à la cour au quotidien, etc ${ }^{8}$.

11 Or ces différents types de production que l'on peut faire entrer également dans le vaste champ de la prose d'idées/littérature d'idées ont également connu un immense succès lors de leur publication manuscrite ou imprimée. Citons seulement deux exemples. Le premier est tiré de la littérature dite économique qui cultive la forme de l'avis, toujours oubliée dans les chapitres sur la prose d'idées de nos littératures ; il s'agit de l'ouvrage de l' «arbitrista " Valle de la Cerda, Avisos en materia de estado y guerra, (imprimés à Madrid, en 1599 chez Pedro Madrigal) dont la traduction française datée de 1629 est due à Anthoine d'Ardilly9. L'autre est celui de Juan Márquez, frère augustin (1565 ?-1621), auteur d'un traité d'éducation du prince El Gobernador cristiano (Celui qui gouverne en chrétien), imprimé en 1612 à Madrid sur une commande en 1604 du duc de Feria vice-roi de Sicile. L'ouvrage qui connut 4 rééditions et 11 impressions successives fut traduit en français par Didier Virion dans une édition imprimée à Nancy en $1621^{10}$. Márquez est également l'auteur d'une histoire de l'ordre des Augustins dont la traduction en Italien par Innocentio Rampini fut imprimée en 1620 à Tortona et d'une biographie d'Alfonso d'Orozco, un religieux de l'ordre, dont le texte fut traduit en italien et publié à titre posthume à Bologne en $1657^{11}$. Ce succès international retentissant pour ce qui concerne Márquez justifie que l'on fasse à ces auteurs la place qu'ils méritent au sein d'une réflexion sur la prose d'idées en Espagne ${ }^{12}$. Outre ces deux exemples, c'est bien sûr une production foisonnante qui entre dans cette catégorie.

La tache est effrayante et passionnante à la fois et l'est d'autant plus qu'elle fait appel à plusieurs disciplines pour son interprétation. Pour cette raison, le matériel utile à l'analyse de ces textes se trouvera non seulement chez les historiens des idées (Thuau, Maravall, Abellán), chez les historiens de la philosophie (Skinner) mais aussi chez les historiens du droit (Hespanha, Clavero). L'intérêt par exemple de la Historia crítica del 
pensamiento español ${ }^{13}$ de José Luis Abellán, qui est un compromis entre histoire des idées et histoire de la philosophie, réside dans le fait que ses analyses associent pour un panorama complet de la période, la pensée des écrivains économiques à la philosophie de Gracián et aux thèmes du théâtre baroque. Mais la logique adoptée laisse de côté les aspects formels des œuvres concernées pour adopter seulement une classification thématique. Par voie de conséquence, José Luis Abellán n'emploie jamais à propos des productions qu'il étudie le terme de prose d'idées.

13 L'intérêt de la prose d'idées pour l'histoire des formes littéraires est pourtant considérable. Nous n'aborderons pas la littérature morale à propos de laquelle la bibliographie est pléthorique. Encore une fois, Quevedo, qui entre autre apport, rénove sur le mode satirique le genre du songe, et Gracián, théoricien de la pointe, y occupent la première place et c'est légitime.

14 Il est néanmoins incontestable que la réflexion sur l'écriture de l'histoire participe non seulement aux bouleversements épistémologiques dont l'époque est porteuse mais contribue également au processus de légitimation de la narration en prose. Prenant racine dans la pensée augustinienne du temps et se nourrissant entre autres de la vogue des biographies laïques inspirées des œuvres morales de Plutarque et des Vies et Doctrines des philosophes illustres de Diogène Laërce mais aussi des Annales de Tacite, l'écriture de l'histoire comporte également une part de réflexivité qui l'associe à part entière à nos préoccupations. On peut citer à cet égard le De historia para entenderla $y$ escribirla de Cabrera de Córdoba ${ }^{14}$.

Par ailleurs et pour se référer seulement à un autre cas, il est établi que la redécouverte du style tacitiste par la littérature politique qui prend pour modèle les Annales de Tacite (ce sont entre autres les œuvres de Baltasar Alamos de Barrientos déjà cité) a fortement contribué à modeler le goût du public pour la forme brève ${ }^{15}$. Les ouvrages de la prose d'idées au XVII ${ }^{e}$ siècle participent donc au foisonnement générique qui caractérise la période et tirent leur légitimité des genres qu'ils adoptent, grâce à leur grande diffusion et à l'audience qu'ils connaissent auprès du lectorat.

16 C'est pourquoi le flou dont on est bien obligé de constater qu'il préside à la définition de la catégorie dans les littératures recouvre en réalité des problèmes d'ordre épistémologique et générique. Les repérer requiert une analyse au cas par cas. L'étude maintenant proposée s'attache à plusieurs aspects d'une des trois œuvres en prose d'Antonio López de Vega: le Songe du parfait seigneur. Songe politique, daté de 1626 et réédité en 1652 et 1653 assorti de "discours variés " et "poèmes divers ». López de Vega est également l'auteur de deux œuvres qui serviront de référence, le cas échéant :

- Heraclite et Démocrite de notre siècle publié en 1641.

- Les Paradoxes rationnels, dont les préliminaires sont datés de 1654, et qui n'ont pas été publiés du vivant de l'auteur.

17 La perspective adoptée envisage les problèmes génériques formulés précédemment en tant qu'ils sont susceptibles d'éclairer la thématique convoquée, c'est-à-dire le message à délivrer. Tout d'abord, insistons sur la réception dont l'œuvre de López de Vega a fait l'objet au cours des siècles parce qu'elle est révélatrice des problèmes de classification posés par la prose d'idées. 


\section{La réception-compréhension de l'œuvre de López de Vega}

18 partie de ces auteurs qui n'apparaissent pas dans les littératures sous la catégorie "prose d'idées ». Encore une fois, la renommée de Lopez de Vega n'est pas en cause puisqu'il a joui d'une audience certaine de son vivant comme en témoignent les trois éditions du Songe du parfait seigneur. L'édition de sa deuxième œuvre en prose, Héraclite et Démocrite de notre siècle, est financée par un homme très influent dans le monde des lettrés de l'époque, le libraire Alonso Pérez, père du poète Alonso Pérez de Montalbán et protecteur du "phénix des esprits », Lope de Vega Carpio ${ }^{16}$. La renommée de López de Vega s'étend jusqu'au XvIII ${ }^{\mathrm{e}}$ siècle puisque malgré leur défiance à l'égard des auteurs baroques, les Lumières espagnoles Mayáns, Capmany, Forner, le classent parmi les auteurs de référence pour la clarté de sa langue et son éloquence ${ }^{17}$. problèmes de classification posés par l'étude de la prose d'idées. Mayáns vante non seulement l'éloquence de Lopez de Vega mais aussi la propriété des termes qu'il emploie, ainsi que la pureté de son style, comprenons son classicisme. C'est le critère rhétorique qui prévaut dans l'appréciation de l'auteur du Songe politique par Mayáns. Pour l'érudit valencien comme pour les critiques de son époque très attachés aux classifications, le classement des œuvres se fait toujours selon les styles: familier, philosophique ou historique, celui de l'oratoire sacré étant le plus éminent. Comme le rappelle François Lopez, au XVIII ${ }^{\mathrm{e}}$ siècle, la fiction cervantine ou celle de Quevedo sont également assimilées à un style. L'appréciation du Quichotte se fonde sur des éléments historiques puisque l'histoire, c'est aussi bien l'invention poétique que le cours des événements passés ${ }^{18}$. Dans le dictionnaire de l'Académie, daté du début du XVIII ${ }^{\mathrm{e}}$ siècle, un des sens de "historia » est bien celui de "fábula o enredo" [fable ou nœud de l'intrigue], le terme de "enredo » étant souvent appliqué au théâtre; ce sens du mot «historia " s'applique à tout phénomène d'imitation d'événements communs ou vraisemblables. Cette définition n'entre donc pas en contradiction avec la citation de Lope de Vega à laquelle recourt le même dictionnaire pour définir la comédie :

Je pense que les romans obéissent aux mêmes règles que les comédies; leur fin commune est bien que leurs auteurs donnent du plaisir et de la joie au peuple même si l'on y sacrifie la manière ${ }^{19}$.

Dans ces propos, roman et comédie, clairement distincts dans l'esprit de leur auteur, n'ont pas d'autre règle que de satisfaire le public qu'ils ont en commun. Texte dramatique et texte narratif sont donc jugés à la même aune, celle de la relation établie entre l'œuvre et son public. Le mode de réception sert donc de critère de définition pour les deux genres. Le pragmatisme du classement opère dans la médiation établie entre l'œuvre et le lecteur.

La réception de López de Vega au XVIII ${ }^{\mathrm{e}}$ siècle pointe inévitablement l'historicité et donc l'instabilité, ou plutôt l'instabilité et donc l'historicité des classifications dont les implications ne peuvent être saisies qu'en un temps donné. On pourrait en dire tout autant de sa réception au $\mathrm{XIX}^{\mathrm{e}}$ et au $\mathrm{XX}^{\mathrm{e}}$ siècle. Au XIX siècle, López de Vega est redécouvert par les grands bibliophiles (Gallardo, García Pérez etc). Ses œuvres retiennent alors l'intérêt de deux grands courants : celui de l'historiographie politique et celui de l'histoire de la littérature.

Cahiers de Narratologie, 14 | 2008 

López de Vega; elle lui a accolé et pour longtemps l'étiquette d'écrivain hétérodoxe (que l'on doit à Marcelino Menéndez Pelayo), d'une taxinomie pour le moins contestable et qui a fait des adeptes ${ }^{20}$.

l'epoque tels Antonio Cánovas del Castillo ou Jerónimo Béker, sont très désireux de l'époque tels Antonio Cánovas del Castillo ou Jerónimo Bécker, sont très désireux de l'hégémonie européenne de la monarchie espagnole. Leur attitude tient à la fois de celle du bibliographe et de celle de l'historien de la philosophie politique. Le très long article de Cánovas sur «Les idées politiques des Espagnols au temps de la maison d'Autriche ${ }^{21}$ » est une véritable mine pour qui s'intéresse à la littérature politique du Xvir siècle mais aussi à la naissance de la science politique au XIX siècle. Cánovas, atteint par le prurit classificatoire du siècle, ordonne, regroupe, étiquette. Mais il le fait avec l'intelligence et l'immense érudition que l'on voit se refléter dans ses écrits. Il range López de Vega - et c'est très intéressant - parmi les auteurs qui ont produit de "ces livres, que l'on peut appeler seulement semi-politiques $»^{22}$. L'explication donnée par Cánovas éclaire le propos qui a priori peut sembler déroutant. Elle montre en tout cas qu'il entrevoit les implications politiques de la philosophie morale du xvII siècle. D'après lui, le Songe du parfait seigneur. Songe politique de López de Vega (le titre dit déjà sa nature), décrit les rapports entre noblesse et pouvoir et instruit le gentilhomme, afin qu'il « puisse évoluer sans faux-pas dans l'entourage du monarque ${ }^{23}$ ». Plus intéressant encore, Lopez de Vega est classé avec Pedro Fernández Navarrete, auteur économique bien connu des spécialistes, et souvent qualifié d' "arbitrista » en tant qu'il réfléchit aux maux de la monarchie et notamment à son dépeuplement. Cánovas non seulement pressent les implications politiques de la philosophie morale mais rassemble sous une même catégorie les auteurs qui s'occupent de l'économie, c'est-à-dire, comme le dit l'étymologie grecque du terme, du gouvernement que ce soit celui de la maison (en l'occurrence la maison noble pour López de Vega) ou de la monarchie dans le cas de Fernández Navarrete. On pourrait y rajouter le gouvernement de soi, puisque le " paradigme de la gouvernementalité ${ }^{4}$ » selon l'expression foucaldienne qui régit la philosophie morale au XVII ${ }^{\mathrm{e}}$ siècle restitue la triple dimension du gouvernement selon un ordre croissant de l'objet, gouvernement de soi (l'éthique), gouvernement de la maison (économie ou économique - on trouve les deux mots au xvII ${ }^{\mathrm{e}}$ siècle), gouvernement de la monarchie (politique). L'article de Cánovas est avant tout le produit d'une époque où il convient de trouver dans le siècle d'or espagnol les racines d'une histoire nationale. Plus subtilement, il manifeste et c'est là son intérêt pour notre propos une intuition des problèmes épistémologiques qui entoure le concept du politique, de l'économique, et du moral au siècle d'or.

Pour terminer ce panorama sur la réception de López de Vega, évoquons rapidement le $\mathrm{xx}^{\mathrm{e}}$ siècle. Grâce aux analyses de José Antonio Maravall dans la deuxième moitié $\mathrm{du} \mathrm{xx}^{\mathrm{e}}$ siècle espagnol qui puise beaucoup de sa matière chez les érudits $d u$ XI $x^{e}$ siècle déjà cités, le champ historiographique et critique se diversifie. Historiens de la littérature, du théâtre en particulier ${ }^{25}$, historiens de l'éducation ${ }^{26}$, de la philosophie ${ }^{27}$, de la noblesse et de la culture nobiliaire ${ }^{28}$ lui font les honneurs de pages substantielles. L'éclectisme de la bibliographie est en premier lieu à l'image de la variété des thèmes abordés dans les œuvres de López de Vega et nous allons réfléchir aux implications formelles de cette variété. 
Plus généralement, l'ensemble de ces références est également le reflet de la multiplication actuelle des problématiques internes à chaque discipline, littéraire, historique, philosophique. La menace est peut-être celle de l'émiettement, nécessaire conséquence de l'extraordinaire foisonnement des objets de la connaissance. Ce panorama montre néanmoins que l'analyse des textes de la prose d'idées a vocation à rassembler. Rassembler plutôt que segmenter les approches propres aux différentes disciplines tout en pointant, quand c'est possible, les problèmes épistémologiques posés par ces rapprochements.

\section{La « Variété » chez López de Vega}

La diversité des disciplines mises en jeu par la prose d'idées tient à un caractère bien spécifique de cette production: la variété. Par ses implications thématiques et formelles, elle explique le caractère difficilement classable de la production de la prose d'idées. Mais la notion de variété permet d'aborder cette inclassabilité à partir d'une notion non forgée a posteriori mais qui est fortement mise en avant par les auteurs du XVII siècle eux-mêmes.

La notion de variété n'est pas ignorée à l'époque concernée. Tout au contraire. Les premiers critiques littéraires qui se sont intéressés aux auteurs du siècle d'or et qui sont encore, on l'aura compris, les érudits du siècle suivant font l'éloge de ceux qui "furent éloquents par la variété de leurs écrits ${ }^{29}$ "- l'expression est due à Gregorio Mayáns ; la variété confondant à la fois la diversité thématique propre à chaque écrit ainsi que l'utilisation alternée ou conjointe de la prose et du vers.

La pratique de la variété chez López de Vega obéit à ce critère de l'éloquence définie par le XvIII ${ }^{e}$ siècle. Elle est extrêmement présente dans son œuvre et repérable dès le seuil des ouvrages. Elle entre donc en jeu au moment de la mise en place dans le paratexte d'une stratégie d'écriture et de la pragmatique qui régit la relation au lecteur.

Du point de vue thématique, l'œuvre de López de Vega est placée sous le signe de l'éclectisme que reflètent les sources bibliographiques antérieurement citées. L'auteur tantôt décrit la figure du parfait gentilhomme et vante les mérites de la noblesse moyenne, tantôt vilipende les praticiens de toutes les disciplines connues (grammaire, poésie, histoire, philosophie naturelle, astrologie, droit, politique, mathématiques) pour à la fin encenser la philosophie morale qu'il fait sienne. Il s'attaque aux mauvais ministres et dénonce les caprices du favori, puis pèse les avantages et les inconvénients du mariage. Il prend part à la polémique sur la nouvelle comédie lopesque puis examine les critères du témoignage historique et de l'expérience de l'événement vécu. Cette variété thématique n'intéresse pas ici pour elle-même mais pour la part de publicité dont elle fait l'objet au seuil des ouvrages. Dans les trois œuvres en prose de López de Vega, la stratégie adoptée est la même. Dès le titre, la variété thématique sert d'effet d'annonce et ce contrat de lecture est renforcé dans la deuxième et la troisième œuvre par la présence en tête de l'ouvrage du sommaire qui rassemble et présente la matière des chapitres composant l'ouvrage. C'est néanmoins la première œuvre de López de Vega qui offre le cas le plus intéressant pour notre propos car la variété thématique y est servie par un éventail non négligeable de formes littéraires. 

trente ans de distance. Le premier, celui de l'édition de 1626, est le suivant : Songe du parfait Seigneur. Songe politique. En 1652, l'édition est augmentée et le titre allongé restitue le contenu ajouté par rapport à l'édition princeps. Le voici : Songe du parfait seigneur. Songe politique assorti de divers discours et de récents poèmes variés. Il est clairement axé sur le thème de la variété, variété des formes littéraires adoptées (songe, discours, poésie), variété du choix proposé à l'intérieur même de ces sections : divers discours, poèmes variés. L'édition de 1652 a bien davantage de succès que la première édition de l'œuvre dans laquelle le songe a été publié seul. L'atteste la deuxième impression en 1653 de l'édition augmentée, financée par le même libraire que la première : Gabriel de León.

Que contient l'ouvrage? Un traité d'éducation du seigneur, trois discours, des poèmes de circonstance dont la chronologie, si on s'y arrête, permet de suivre presque pas à pas les événements importants de la vie courtisane sous le règne de Philippe IV. Le dédicataire de l'œuvre, le duc d'Alburquerque, Grand d'Espagne, Gentilhomme de la chambre du roi et futur Vice-roi de Nouvelle Espagne en est un acteur en pleine ascension. Dans sa dédicace au Duc, López de Vega exalte encore une fois la variété de ses discours et de ses vers grâce à laquelle il entend procurer "un honnête divertissement » à son noble lecteur et protecteur. La dédicace se construit de façon très conventionnelle autour du lieu commun cicéronien de l'utile et de l'agréable comme la plupart des textes liminaires de l'époque, tous genres confondus. La question du dédicataire est néanmoins centrale pour notre propos car c'est elle qui confère à l'ouvrage son unité par-delà la variété formelle constatée. Si le Songe du parfait seigneur reprend la forme éprouvée du songe et doit beaucoup à l'ouvrage espagnol fondateur du genre, la Vision delectable d'Alfonso de la Torre datée de $1450^{30}$, son contenu en fait réellement un manuel d'éducation du gentilhomme courtisan. Il emprunte la forme du récit onirique de la Vision delectable et la narration en première personne que le roman picaresque a mis au goût du jour. Il assimile donc les formes du récit à succès qu'il met au service d'une morale en cours d'élaboration en Espagne, celle de la vie de cour. Il garde par ailleurs la cohérence du traité par son ordonnancement en chapitres et ses sous-titres très indicatifs de leur contenu. Le recueil de poèmes de circonstances dont nous avons déjà parlé et qui est ajouté à l'édition de 1652 devient donc un prolongement logique du Songe. Cette sorte de journal en forme poétique de la vie courtisane complète, dans le dispositif de l'ouvrage, les conseils sur la manière de se comporter à la cour prodigués au parfait seigneur. Par ailleurs, un des trois discours ajouté, intitulé discours économique et qui traite d'une réforme menée probablement par le duc d'Alburquerque en sa maison ${ }^{31}$, parfait la mission de conseiller du seigneur que s'est assignée López de Vega tout au long de son ouvrage. Cela nous éclaire sur la portée de l'œuvre et sur l'attitude de l'écrivain à une époque donnée. Le discours économique et les poèmes ont valeur informative, et nous permettent de comprendre le changement d'attitude des auteurs à l'égard de leur lectorat dans la prose d'idées. Ils sont dispensateurs de savoir mais aussi d'information, cette valeur plus que tout prisée à un moment où la culture de l'écrit est en train de prendre une place démesurée dans la vie juridique (il faut prouver par l'écrit), dans la vie du royaume (c'est le moment de la création des archives du royaume ${ }^{32}$ ), dans la vie quotidienne par la multiplication des correspondances. 
tte cohérence éminemment politique et courtisane (mais en espagnol à cette époque-là " politico » signifie politique et courtisan) découle de l'économique au sens où nous l'avons précédemment défini. Le gouvernement de la maison noble, ses règles et ses modèles servent d'épreuves politiques à qui aspire à de plus haute fonction comme c'est le cas du protecteur de López de Vega. Sous la plume de López de Vega, la prose d'idées, dans sa variété divertissante mais puissamment signifiante, devient le faire valoir d'un écrivain dont le statut à l'époque est encore profondément courtisan, au service d'une élite qui a parfaitement compris la force de frappe des moyens de l'écrit. L'interdépendance du politique, de l'économique et de l'éthique est constitutive de cette littérature que l'on ne peut réduire à son qualificatif habituel de littérature politique. Elle ne peut donc qu'entrer de plein droit dans le vaste champ de la prose d'idées, dont les implications formelles et thématiques, et les enjeux épistémologiques font toute la richesse. L'exemple de l'œuvre de López de Vega qui en est un exemple parmi bien d'autres montre que dans la prose d'idées à l'âge baroque, vouloir séparer les différents savoirs mis en jeu revient à encourir le même reproche qu'adressait Gilles Deleuze à Descartes à quelques siècles de distance : « chercher le secret du continu dans les parcours rectilignes et celui de la liberté dans la rectitude de l'âme en ignorant autant l'inclinaison de l'âme que la courbure de la matière ${ }^{33}$ ".

\section{NOTES}

1. La veine du roman byzantin s'inspire des romans grecs d'Héliodore (Les Éthiopiques, et Théagène et Chariclée) et d'Achille Tatius (Leucippé et Clitophon). On en trouve les derniers échos dans Persiles et Ségismonde de Cervantès où le romancier dit vouloir rivaliser avec le maître du genre : Héliodore. Cf. Christine Marguet, «Le roman byzantin espagnol (XVI ${ }^{\mathrm{e}}$-XVII ${ }^{\mathrm{e}}$ siècles) entre plaisir de la lecture et didactisme ", thèse soutenue en 1998 sous la direction d'Augustin Redondo à Paris-III publiée sous le titre Le Roman d'aventures et d'amour en Espagne, XVI $I^{e}$-XVII ${ }^{e}$ siècles. L'utile et l'agréable, Paris, L'Harmattan, 2004.

2. Le roman pastoral se caractérise par le passage au premier plan d'un auteur narrateur personnage, autour duquel s'organise un récit à la première personne du singulier. L'héroïsation de l'univers bucolique (passage des bergers-poètes aux princes) constitue un des axes majeurs du roman pastoral. Cf. Françoise Lavocat, « Princes et poètes en Arcadie : le roman pastoral en Italie, en Espagne et en France de la renaissance du genre à sa décadence : son rôle dans la transformation du roman ", thèse soutenue en 1993 à Paris-VII publiée sous le titre Arcadies malheureuses. Aux origines du roman moderne, Paris, Honoré Champion, 1998.

3. Gutiérrez de los Ríos accorde un place importante aux mathématiques. On considère son œuvre comme une vulgarisation de la pensée de Galilée et de Descartes. Quelques pages lui sont consacrées par José Antonio Maravall « Preludio a la ilustración : "El Hombre práctico" (1680) de Gutiérrez de los Ríos », dans Francisco Rico (dir.), «Historia y crítica de la literatura española », t. 3, coord. Bruce W. Wardropper, Barcelona, Crítica, 1983, 975-981.

4. La traduction française par « arbitriste » n'est pas attestée ; on lui préfèrera celle de «faiseurs d'avis » ou de «donneur d'avis ». L'arbitrista dans l'Espagne du premier XVII ${ }^{\mathrm{e}}$ siècle réfléchit aux maux de la monarchie et propose, moyennant rétribution, des solutions fiscales ou autres pour 
faire accroître la trésorerie du roi. C'est une figure si emblématique du temps qu'il devient un type littéraire tourné fréquemment en dérision dans la littérature satirique du siècle d'or. Voir Jean Vilar, Literatura y Arbitrismo. La Figura satírica del arbitrista en el siglo de oro, Madrid, Revista de occidente, 1973.

5. Ils sont cités par Raphaël Carrasco, «La prose, de Quevedo a Gracián », in Jean Canavaggio (dir.), Histoire de la littérature espagnole, Paris, Fayard, 1993, t. 1, p. 710-723.

6. Maurice Molho, «Quevedo", Jean Canavaggio (dir.), Histoire de la littérature espagnole, t. 1, Paris, Fayard, 1993, p. 657.

7. El Oráculo manual y arte de prudencia (1647) a été traduit par L'Homme de cour. Traduit de l'espagnol de Balthasar Gracian, par le sieur Amelot de La Houssaie, Paris, Veuve Martin et J. Boudot, 1684. Cette traduction a connue 6 éditions successives entre 1684 et 1696 et a constamment été rééditée tout au long $\mathrm{du} \mathrm{XVIII}^{\mathrm{e}}$ siècle. Éditions récentes de cette traduction, Baltasar Gracian, L'Homme de cour, trad. Amelot de La Houssaie, Paris, Ivrea, 1993. Édition espagnole en ligne établie a partir de l'édition de Huesca, Juan Nogués, 1647 et de l'édition critique d'Emilio Blanco (Madrid, Cátedra, 1997) : http://www.cervantesvirtual.com/servlet/SirveObras/ 12693841220138387204735/p0000001.htm.

On attribue également à Amelot de la Houssaye, la traduction de El Comulgatorio (1655), Modèle d'une sainte et parfaite communion en 50 méditations... pour tous les dimanches et les festes de l'année,... traduites de l'espagnol de Baltazar Gracian [par A.-N. Amelot de La Houssaye], Paris, J. Boudot, 1693. édition espagnole modernisée en ligne du texte de 1655, à partir de l'édition d'Evaristo Correa Calderón (Gracián, Baltasar, El comulgatorio, Madrid, Espasa-Calpe, 1977) : http:// www.cervantesvirtual.com/servlet/SirveObras/23582846543469417965679/p0000001.htm\#I_0_

8. Sur le sujet, Augustin Redondo, (dir.), Las Relaciones de sucesos (canards) en Espagne (1500-1750). Actes du premier colloque international (Alcalá de Henares, 8, 9 et 10 juin 1995), Paris, Publications de La Sorbonne et Universidad de Alcalá, Travaux du CRES, n XII, 1996 ; Sagrario López Poza y Nieves Pena Sueiro (éd.), La Fiesta. Actas del II Seminario de Relaciones de Sucesos. (A Coruña, 13-15 de julio de 1998), Ferrol, Sociedad de cultura Valle Inclán, 1998. Voir les travaux de la Sociedad Internacional para el Estudio de las Relaciones de Sucesos (SIERS) en Espagne dirigée par Sagrario López Poza, http://rosalia.dc.fi.udc.es/relaciones/. Publications recensées à la page: http:// rosalia.dc.fi.udc.es/SIERS/publicaciones.html.

9. Une explication sur le terme d'arbistrista pour les non initiés : en français on peut traduire " arbitrista » par donneur d'avis. Ils sont des faiseurs d' " arbitrios », c'est-à-dire des hommes qui proposent au roi des solutions d'ordre économique ou fiscale pour alléger sa dette. Sociologiquement ils sont souvent juristes généralement issus de l'administration du roi. Très écoutés durant les sessions des Cours (des cortes) du royaume, leurs propositions font généralement l'objet d'une publication à compte d'auteur ou financées par les Cours elles-mêmes. Du point de vue thématique, ils dénoncent toujours les maux dont souffrent le royaume et la monarchie afin que leurs propositions, dont la mise en œuvre est généralement soumise à leur rémunération préalable, soient perçues comme providentielles.

10. Traduction française de Didier Virion, Seigneur de They publié à Nancy en 1621 sous le titre L'Homme d'Estat chrestien.

11. Origen de los frayles ermitaños de la orden de san Augustín, Salamanca, A. Ramirez, 1618, traduction italienne de Rampini, Origine delli frati eremitani dell' ordine di S. Agostino, Tortona, N. Viola, 1620. Vida deSan Alonso de Orozco, Madrid, J. Sánchez, 1648, trad. de Luigi Torelli, Vita del venerabil servo di Dio F. Alfonso d'Orosco, Bologna, 1657.

12. Anne Dubet a soutenu une thèse sur Valle de la Cerda publiée sous le titre, "Réformer les finances espagnoles au Siècle d'Or. Le projet Valle de la Cerda", Clermont-Ferrand, Presses universitaires Blaise-Pascal, 2000. Thèse de Francisco Javier López de Goicoechea Zabala, sur la pensée politique de Juan Márquez, publiée sous le titre, Juan Márquez: un intelectual de su tiempo, Madrid, Revista Agustiniana, 1996. 
13. Madrid, Espasa-Calpe, 1979-1991. Particulièrement, Del barroco a la ilustración, vol. 3.

14. Voir Renaud Malavialle, L'Essor de la pensée historique au siècle d'or. De Juan Luis Vives à Antonio de Herrera y Tordesillas, thèse de doctorat sous la direction de Jean-Michel Laspéras, université de Provence, 2003, et l'intervention de l'auteur dans le séminaire "Récit et genres historiques ", Jean-Paul Aubert et Aude Déruelle (coord.) dont les interventions seront publiées dans un volume de cette revue électronique.

15. Mercedes Blanco, dans son travail sur le style de Gracián, associe la filiation entre le style tacitiste et l'art de la pointe dans l'œuvre du grand moraliste : La Rhétorique de la pointe. Gracián et le conceptisme, Paris, Champion, 1992.

16. Anne Cayuela a récemment donné une étude de l'inventaire de sa librairie: Alonso Pérez de Montalbán. Un librero en el Madrid de los Austrias, Madrid, Calambur, 2005.

17. Sur la réception de López de Vega au XVIII siècle, voir Marie-Laure Acquier, « Réflexions sur le lien entre les penseurs baroques et les Lumières. Le cas d'Antonio López de Vega (1586?-1656?) », in Christophe Couderc et Benoît Pellistrandi (coord.), " Por discreto y por amigo ». Mélanges offerts à Jean Canavaggio, Madrid, Casa de Velásquez, 2005, p. 473-485.

18. François Lopez, "La institución de los géneros literarios en la España del siglo XVIII », Bulletin hispanique, t. 102, $n^{\circ}$ 2, juillet-décembre 2000, p. 486.

19. Ibid., p. 473-517. La traduction de Lope de Vega est de mon fait.

20. Voir l'article de Henry Méchoulan, «Fadrique Furio Ceriol et Antonio López de Vega, deux hétérodoxes méconnus », publication de l'université de Toulouse-Le Mirail, «Penseurs hétérodoxes du monde hispanique, 1974, pp. 115-132».

21. Antonio Cánovas del Castillo, «Las ideas políticas de los Españoles en tiempos de la Casa de Austria ", paru en deux parties dans la Revista de España, première partie, t. IV, 1868, p. 497-570, deuxième partie, t. VI, 1869, p. 40-99.

22. " estos libros, que se pueden llamar solo semi-políticos », Ibid., t. IV, p. 553-554.

23. Ibid.

24. Michel Foucault, « La gouvernementalité », Dits et écrits. 1954-1988, Paris, Gallimard, 1994, t. 2, p. 635-657.

25. Marc Vitse, Éléments pour une théorie du théâtre espagnol du XVII siècle, Toulouse, Presses universitaires du Mirail, 1990, Margarete Newels, Los Géneros dramáticos en las poéticas del siglos de oro, Londres, Támesis, 1974.

26. Jean-Michel Laspéras, "Manuales de educación en el siglo de oro ", Bulletin Hispanique, t. 97, $\mathrm{n}^{\circ}$ 1, 1995, p. 173-185, du même auteur, «Antonio López de Vega et l'éducation : le temps des ruptures », in Jean-Louis Guereña, Famille et Education en Espagne et en Amérique Latine, Actes du colloque de Tours, publications de l'université François Rabelais de Tours, série «Etudes hispaniques ", n XV-XI, 2002, p. 467-480 ; Carlos Vaíllo, " La formation culturelle de la personne chez Antonio López de Vega ", in La Transmission du savoir dans l'Europe des XVI et XVII ${ }^{e}$ siècles, actes du colloque de Nancy, 20-22 novembre 1997, Paris, Champion, 2000, p. 69-79.

27. José Luis Abellán, Historia crítica del pensamiento español, vol. 3, op. cit.; Karl Alfred Blüher, Séneca en España. Investigaciones sobre la recpeción de Séneca en España des el siglo XIII hasta el siglo XVII, Madrid, Gredos, 1983 ; Jeremy Robbins, «Scepticism and Stoïcism in Spain : Antonio López de Vega's Heraclito y Democrito de nuestro siglo ", in P. Thompson, Eric Southworth, Clive Griffin, Nigel Griffin (coord.), “Culture and Society in Habsburg's Spain : Studies Presented to R. W. Truman by his Pupils and Colleagues on The Occasion of his Retirement", Londres, Tamesis, Serie A, Monografías 190, 2001, p. 137-152.

28. Ignacio Atienza Hernández, « El señor avisado: programas paternalistas y control social en la Castilla del siglo XVII ", Manuscrits. Revista d'història moderna, n 9, 1991, p. 155-204 ; David García Hernán, La Nobleza española en la edad moderna, Madrid, ISTMO, 1992. Charles Jago, “ La 'crisis de la aristocracia' en la Castilla del siglo XVII ", in J. H. Elliott, (éd.), Poder y Sociedad en la España de los Austrias, Barcelone, Crítica, 1982, p. 249-286. 
29. "elocuentes en la variedad de sus escritos", Gregorio Mayáns, Oración en alabanza de las eloquentíssimas obras de Don Diego de Saavedra Fajardo, 1725 dedicado al marqués de Villena, fundador y director de la Real Academia Española, citado por François Lopez, ibid. p. 479.

30. L'ouvrage d'Alfonso de la Torre associe de façon définitive étant donné son succès la forme du songe et la philosophie morale. Lopez de Vega emprunte cette heureuse association à un auteur à succès.

31. C'est l'objet de la démonstration de Charles Jago, loc. cit.

32. Charles Quint ordonne le transfert des premières archives du royaume dans la forteresse de Simancas par une cédule royale du 16 septembre 1540. Mais c'est son fils Philippe II qui donnera son véritable statut d'archives de la couronne à la forteresse de Simancas par des Instructions dités en 1588. C'est le premier règlement des archives.

33. Gilles Deleuze, Le Pli, Paris, Minuit, 1988, p. 5.

\section{RÉSUMÉS}

Dans le domaine hispanique, la prose d'idées souffre de façon endémique d'un déficit de définition. Dans les histoires de la littérature espagnole, elle apparaît comme une catégorie où sont rassemblées des productions textuelles difficilement classables appartenant à l'Espagne de l'époque moderne. Ces productions sont d'autant moins classables qu'elles voient le jour à une période caractérisée par une double indétermination: les champs disciplinaires y sont en gestation et les genres littéraires en cours d'élaboration. L'œuvre du polygraphe Antonio López de Vega dans sa diversité générique et thématique nous offre un exemple de ce qu'était la prose d'idées au XVIIe siècle, une littérature qui se donnait à lire comme productrice de savoirs.

En el ámbito hispánico, la prosa de ideas suele padecer de un defícit de definición. En las historias de la literatura española, aparece como una categoría donde se juntan producciones textuales difíciles de clasificar y que pertenecen a la España de la época moderna. Dichas producciones son tanto menos clasificables cuanto que salen a luz en un periodo afectado por una doble indeterminación: los campos de aplicacion de las disciplinas del saber están en curso de elaboración así como los géneros literarios. La obra del polígrafo Antonio López de Vega por su diversidad genérica y temática ofrece un ejemplo de los que era la prosa de ideas en el siglo XVII, una literatura que producía un saber.

\section{INDEX}

Index géographique : Espagne

Index chronologique : XVIIe siècle

Mots-clés : Espagne du XVIIe siècle, genres et savoirs, prose d'idées 
AUTEUR

\section{MARIE-LAURE ACQUIER}

Agrégée d'Espagnol, ancien membre de la Casa de Velázquez de Madrid, Marie-Laure Acquier est maître de conférences à l'Université de Nice-Sophia antipolis. 